\title{
Clinical and Serological Associations with the Development of Incident Proteinuria in Danish Patients with Systemic Lupus Erythematosus
}

\author{
Nima Tanha, Renata Baronaite Hansen, Christoffer Tandrup Nielsen, Mikkel Faurschou, \\ and Søren Jacobsen
}

\begin{abstract}
Objective. In a longitudinal cohort study, we investigated whether clinical and serological manifestations at the time of classification of systemic lupus erythematosus (SLE) were predictive of subsequent development of incident proteinuria as a biomarker of incident lupus nephritis.

Methods. Patients fulfilling SLE classification criteria but having no proteinuria prior to or at the time of classification were included. Data on SLE manifestations, vital status, criteria-related autoantibodies, and SLE-associated medications were collected during clinical visits and supplemented by chart review. HR were calculated by Cox regression analyses.

Results. Out of 850 patients with SLE, 604 had not developed proteinuria at the time of SLE classification. Of these 604 patients, 184 (30\%) developed incident proteinuria following SLE classification. The patients had a median followup of 11 years and 7 months. Younger age and history of psychosis at the time of classification were associated with development of incident proteinuria, just as were lymphopenia (HR 1.49, 95\% CI 1.08-2.06), anti-dsDNA (HR 1.38, 95\% CI 1.01-1.87), and a high number of autoantibodies (HR 1.26, 95\% CI 1.06-1.48).

Conclusion. The risk of incident proteinuria after onset of SLE was increased by the presence of lymphopenia, anti-dsDNA antibodies, psychosis, younger age, and a high number of autoantibodies at onset. (First Release April 15 2018; J Rheumatol 2018;45:934-41; doi:10.3899/jrheum.170933)
\end{abstract}

Key Indexing Terms:

SYSTEMIC LUPUS ERYTHEMATOSUS

AUTOANTIBODIES

\section{LUPUS NEPHRITIS LYMPHOPENIA}

Systemic lupus erythematosus (SLE) is a systemic autoimmune disease with diverse clinical manifestations and a course of illness ranging from benign forms with limited organ involvement to potentially fatal multiorgan involve-

From the Copenhagen Lupus and Vasculitis Clinic, Center for

Rheumatology and Spine Diseases, Rigshospitalet, Copenhagen University Hospital, Denmark.

Supported by The Danish Rheumatism Association (SJ-A3865) and The Board of Research at The Copenhagen University Hospital, Rigshospitalet, Denmark. Funders supported the project financially only and had no influence on the study or reporting of its results.

N. Tanha, MD, Research fellow, Copenhagen Lupus and Vasculitis Clinic, Center for Rheumatology and Spine Diseases, Rigshospitalet, Copenhagen University Hospital; R.B. Hansen, MD, Research Fellow, Copenhagen Lupus and Vasculitis Clinic, Center for Rheumatology and Spine Diseases, Rigshospitalet, Copenhagen University Hospital; C.T. Nielsen, MD, PhD, Copenhagen Lupus and Vasculitis Clinic, Center for Rheumatology and Spine Diseases, Rigshospitalet, Copenhagen University Hospital; M. Faurschou, MD, PhD, Copenhagen Lupus and Vasculitis Clinic, Center for Rheumatology and Spine Diseases, Rigshospitalet, Copenhagen University Hospital; S. Jacobsen, MD, Professor, Copenhagen Lupus and Vasculitis Clinic, Center for Rheumatology and Spine Diseases,

Rigshospitalet, Copenhagen University Hospital.

Address correspondence to Prof. S. Jacobsen, Section 4242 Rigshospitalet, Blegdamsvej 9, DK-2100 Copenhagen, Denmark.

E-mail: sj@dadlnet.dk

Accepted for publication January 26, 2018. ment ${ }^{1}$. Lupus nephritis (LN) is a severe manifestation of SLE associated with a risk of terminal renal failure and mortality ${ }^{2}$. Increased knowledge of early risk factors for $\mathrm{LN}$ is therefore desirable.

Several demographic factors such as male sex ${ }^{3,4}$, early onset $^{5,6}$, and certain ethnicities such as Asian ${ }^{7}$, Mestizo $^{8}$, Hispanic, and African descent ${ }^{9}$ have been associated with a higher risk of developing LN. However, studies of early SLE-related clinical and serological manifestations as predictors for the development of LN among patients with SLE are scarce. A recent study of predictors of incident proteinuria among patients with SLE confirms the significance of age and serological manifestations for the development of incident proteinuria after SLE diagnosis ${ }^{10}$. The aim of our study was to corroborate and expand on these findings with a focus on manifestations at the time of SLE diagnosis; these manifestations may predict development of subsequent incident proteinuria in nonrenal SLE.

\section{MATERIALS AND METHODS}

In this longitudinal study, data were collected on all inpatients and outpatients with SLE seen at the Department of Rheumatology, Rigshospitalet, in Copenhagen between 1995 and 2015. In addition, data on patients with SLE seen between 1975 and 1995 were collected from several nationwide

Personal non-commercial use only. The Journal of Rheumatology Copyright ()$_{2018}$. All rights reserved. 
SLE specialized centers, as previously described ${ }^{11}$. All patients included in our study fulfilled established classification criteria for SLE ${ }^{12,13}$ and the date of diagnosis was defined as the time when the American College of Rheumatology (ACR) classification criteria were first fulfilled.

SLE manifestations, vital status, criteria-related autoantibodies, and SLE-associated medications were collected during clinical visits and supplemented by chart review. Our SLE cohort data reside in DANBIO, a nationwide clinical quality database that provides data on the disease course of patients with inflammatory rheumatic diseases, including SLE ${ }^{14}$. Data were extracted from DANBIO, consolidated, and made available for analysis.

Clinical definitions, outcomes, and exposure variables. Incident proteinuria, the main outcome variable of our study, was defined as having $\geq 2$ measurements of elevated urine protein $(>0.5 \mathrm{~g} / \mathrm{d})$ in the context of SLE. The standard routine of the clinic was that a 24-h urine sample was collected and analyzed for all SLE patients with a positive urine dipstick for proteins. Patients with proteinuria close to $0.5 \mathrm{~g} / \mathrm{d}$ underwent a repeated 24-h urine collection. Because most patients seen in the clinic are referred from other health institutions, a 24-h urine collection was repeated to verify any proteinuria findings from the referring institution.

The SLE-related manifestations studied were all defined by the 1997 ACR revised criteria for the classification of SLE, with the exception of the false-positive syphilis test ${ }^{13}$. The presence of a false-positive syphilis test was excluded as an exposure variable because the test was not routinely used for all patients in our cohort. In addition, alopecia and positive Coombs test, as defined by the Systemic Lupus International Collaborating Clinics criteria $^{15}$, were also examined. All manifestations occurring up to 1 month following the time of classification were regarded as present at the time of diagnosis to take into account any delays in ordered laboratory results or clinical data reporting. The number of autoantibodies was defined as the sum of any of the following positive laboratory findings: anti-dsDNA antibodies, antinuclear antibodies (ANA), anti-Sm antibodies, lupus anticoagulant (LAC), immunoglobulin (Ig-) $\mathrm{G}$ anticardiolipin (aCL), and IgM aCL antibodies.

Autoantibodies were measured by routine methods used for the currently applied clinical practice (i.e., ANA was typically determined by immunofluorescence methods and the other autoantibodies by ELISA). LAC was typically determined by a mixing test of patient plasma with normal pooled plasma. The dilute Russell's viper venom time was used to confirm diagnosis in the case of presence of clotting inhibitor in the mixing test.

Other exposure variables studied were age, sex, prodromal time, hypertension, and SLE-related medications taken prior to or at the time of diagnosis. Prodromal time was defined as the time from the first occurrence of any of the manifestations studied until time of diagnosis.

Date of hypertension was defined as the time of initiation of the first antihypertensive drug treatment. SLE-related medications were glucocorticoids (oral, intravenous, subcutaneous, intramuscular), antimalarials (hydroxychloroquine), and immunosuppressants (i.e., azathioprine, cyclophosphamide, cyclosporine, methotrexate, rituximab, and mycophenolate mofetil).

The cohort was followed through December 2016. Followup time was defined as time from date of diagnosis (i.e., classification) until the last hospital visit or death, whichever came first.

Statistical analysis. Statistical analyses using SPSS Statistics 22.0 software (IBM) included Kaplan-Meier plotting and Cox proportional hazards regression analyses. Cox regression analyses were used to analyze the risk of developing incident proteinuria after diagnosis of nonrenal SLE, relative to the previously explained exposure variables at the time of diagnosis. Cox regression analyses were conducted using univariate analyses and combined models adjusting for all variables statistically significant in the univariate analyses. Two multivariate combined models were conducted, selecting anti-dsDNA for model 1 and number of autoantibodies for model 2 because of redundant information in these 2 serological variables. HR are presented with their corresponding $95 \%$ CI. Plots of survival free of proteinuria were generated based on Kaplan-Meier estimates. Differences and trends between strata were analyzed by means of the log-rank test. Statistical significance was defined as $\mathrm{p}<0.05$.

Ethics statement. DANBIO has previously been approved by the Danish Data Registry (J. nr. 2007-58-0014 and J. nr 2007-58-0006) and the National Board of Health (J. nr. 7-201-03-12/1).

\section{RESULTS}

The recruitment base for our study consisted of 850 patients. Their characteristics are detailed in Table 1 . There were 246 (29\%) patients who had proteinuria prior to or at the time of diagnosis. A flowchart illustrating the distribution of proteinuria among the patients with SLE at the time of diagnosis and during followup is shown in Figure 1.

There were 604 patients at risk of developing incident proteinuria, and about $30 \%$ of these patients (184 patients) developed proteinuria by the end of followup. Seventeen (2.8\%) patients had developed endstage renal disease and 112 had died by the end of followup (data not shown). Clinical characteristics, demographics, and SLE-related medications taken at the time of and/or prior to the time of SLE diagnosis for the 604 included patients are presented in Table 1.

The associations between exposure variables at time of diagnosis and subsequent incident proteinuria are shown as HR in Table 2. Incident proteinuria during followup was associated with lymphopenia (HR 1.49, 95\% CI 1.08-2.06) and anti-dsDNA (HR 1.38, 95\% CI 1.01-1.87). Because some patients were receiving medications that could affect the lymphocyte count, the use of SLE-related medications at the time of diagnosis was adjusted for in a separate Cox regression analysis. Medication usage at the time of diagnosis in this separate analysis was defined as any SLE medication prescribed during 6 months prior to and at the time of diagnosis. In this same analysis, lymphopenia was still significantly associated with incident proteinuria (data not shown). Figure 2 shows that lymphopenia was associated with survival free of incident proteinuria $(\mathrm{p}=0.028$ by log-rank test). For each increase in number of autoantibodies at the time of diagnosis, the risk of developing proteinuria was increased by a factor of 1.26 (95\% CI 1.06-1.48). Patients with $\geq 4$ autoantibodies at the time of diagnosis had 2.15-times (95\% CI: 1.09-4.21) increased risk of developing proteinuria compared to patients with $\leq 1$ autoantibody present at the time of diagnosis. Figure 3 shows how decreasing survival free of proteinuria was associated with an increasing number of autoantibodies at diagnosis ( $\mathrm{p}=0.017$ by log-rank test). Patients with SLE $<40$ years old had about 3-times increased risk of incident proteinuria compared to patients $>60$ years at the time of diagnosis. In a separate analysis, a 1-way ANOVA test was conducted and showed no significant difference in the mean followup time of the different age groups (data not shown). Prodromal time was not associated with development of incident proteinuria. A history of psychosis at the time of diagnosis was also associated with development of incident proteinuria, which mainly occurred during the first years of

Personal non-commercial use only. The Journal of Rheumatology Copyright (c) 2018. All rights reserved. 
Table 1. Demographic and clinical characteristics of patients with SLE at the time of diagnosis. Values are n (\%) unless otherwise specified.

\begin{tabular}{|c|c|c|}
\hline Characteristics & $\begin{array}{l}\text { All SLE Patients, } \\
\qquad \mathrm{n}=850\end{array}$ & $\begin{array}{l}\text { SLE Patients at Risk } \\
\text { of Developing Proteinuria, } \\
n=604\end{array}$ \\
\hline \multicolumn{3}{|l|}{ Sex } \\
\hline Male & $104(12)$ & $56(9.0)$ \\
\hline Female & $746(88)$ & $548(91)$ \\
\hline \multicolumn{3}{|l|}{ Age, yrs } \\
\hline Median & 32 & 34 \\
\hline Range & $3-81$ & $3-81$ \\
\hline \multicolumn{3}{|l|}{ Prodromal time, yrs } \\
\hline Median & 1.5 & 1.8 \\
\hline Range & $0-44$ & $0-44$ \\
\hline \multicolumn{3}{|l|}{ Followup time, yrs* } \\
\hline Median & 10 & 12 \\
\hline Range & $0-44$ & $0-44$ \\
\hline \multicolumn{3}{|c|}{ Clinical manifestations, cumulative } \\
\hline Malar rash & $354(42)$ & $263(44)$ \\
\hline Discoid rash & $83(9.8)$ & $74(12)$ \\
\hline Photosensitivity & $252(30)$ & $219(36)$ \\
\hline Oral ulcers & $83(9.8)$ & $67(11)$ \\
\hline Arthritis & $516(61)$ & $397(66)$ \\
\hline Pericarditis & 107 (13) & $77(13)$ \\
\hline Pleurisy & $198(23)$ & $143(24)$ \\
\hline Psychosis & $20(2.4)$ & $18(2.9)$ \\
\hline Seizure & $25(2.9)$ & $15(2.5)$ \\
\hline Leukopenia & $184(22)$ & $130(22)$ \\
\hline Lymphopenia & $252(30)$ & $168(28)$ \\
\hline Thrombocytopenia & $196(23)$ & $127(21)$ \\
\hline Hemolysis & $67(7.9)$ & $39(6.5)$ \\
\hline Anti-dsDNA & $455(54)$ & $343(57)$ \\
\hline Anti-Sm & $38(4.5)$ & $25(4.1)$ \\
\hline IgG or IgM cardiolipin & $186(22)$ & $126(21)$ \\
\hline LAC & $29(3.4)$ & $19(3.1)$ \\
\hline ANA & $796(94)$ & $565(94)$ \\
\hline Alopecia & $100(12)$ & $72(12)$ \\
\hline Positive Coombs test & $122(14)$ & $74(12)$ \\
\hline Hypertension & $87(10)$ & $25(4.1)$ \\
\hline \multicolumn{3}{|l|}{ SLE-related medication } \\
\hline Glucocorticoids & $540(64)$ & $340(56)$ \\
\hline HCQ & 333 (39) & $276(46)$ \\
\hline Immunosuppressants & $293(35)$ & $150(25)$ \\
\hline AZA & $156(18)$ & $102(17)$ \\
\hline CYC & $76(8.9)$ & $13(2.2)$ \\
\hline CSA & $12(1.4)$ & $5(0.8)$ \\
\hline MTX & $50(5.9)$ & $44(7.2)$ \\
\hline MMF & $56(6.6)$ & $14(2.3)$ \\
\hline RTX & $8(0.9)$ & $4(0.7)$ \\
\hline
\end{tabular}

* Followup time/SLE duration: time from date of diagnosis (i.e., fulfillment of the ACR criteria) until last hospital visit or death, whichever came first. SLE: systemic lupus erythematosus; ACR: American College of Rheumatology; IgG: immunoglobulin G; LAC: lupus anticoagulant; ANA: antinuclear antibodies; HCQ: hydroxychloroquine; AZA: azathioprine; CYC: cyclophosphamide; CSA: cyclosporine; MTX: methotrexate; MMF: mycophenolate mofetil; RTX: rituximab.

disease (data not shown). A history of antimalarial treatment prior to or at the time of diagnosis was associated with lower risk of proteinuria only in the univariate analysis and not in any of the combined models.

\section{DISCUSSION}

In our present study, anti-dsDNA antibodies, history of psychosis and lymphopenia, younger age, and a high number of autoantibodies at time of diagnosis were found to be associated with the development of incident proteinuria in patients with nonrenal onset of SLE. The findings on the presence of anti-dsDNA and younger age inferring higher risk of developing $\mathrm{LN}$ are in line with a recent study in the United States ${ }^{10}$. However, in that study, anti-Sm antibodies, hypertension, and antimalarial drug treatment were also found to be associated with LN. In our select group of patients with SLE, we did not find such associations with incident proteinuria. However, in that study ${ }^{10}$, anti-Sm antibodies, hypertension, and antimalarial drug treatment were also found to be associated with LN. We did not find such associations with incident proteinuria in our select group of patients with SLE. Another previous US study concluded that, in line with our findings, anti-dsDNA inferred a higher risk of incident $\mathrm{LN}$, and that anti-Sm was not associated with $\mathrm{LN}^{16}$. Interestingly, in another study of Bahraini patients, the presence of anti-Sm was even inversely associated with development of $\mathrm{LN}^{17}$. These discrepant findings are not yet explained, but differences in ethnicity could be a potential source of variation. Further, we found that an increase in the number of autoantibodies at the time of diagnosis inferred a higher risk of developing proteinuria, probably reflecting a composite risk of the above-studied autoantibodies or the level of immunological activity. This trend is clearly demonstrated in the survival plot in Figure 3. To our knowledge, no other studies have investigated trends in the cumulative number of autoantibodies as a specific risk factor for the development of incident proteinuria. However, a previous study from 2005 investigating the role of antibody clustering in SLE found that patients with SLE who were seropositive for LAC, anti-dsDNA, and aCL antibodies were at higher risk of developing LN compared to patients with other serological presentations $^{18}$.

A typical characteristic of $\mathrm{LN}$ is the deposition of intrarenal immune complexes that cause complement activation, inflammation, and subsequent damage to the kidneys ${ }^{2}$. Active LN is, in particular, characterized by the presence of anti-dsDNA IgG antibodies, participating either directly or indirectly in renal damage 19,20,21. Our study indicates that these processes are not only active around the onset of SLE but may also occur during the course of disease in patients with nonrenal onset SLE.

Although relatively many studies have investigated the role of autoantibodies in SLE and subsequent LN, there are few studies concerning lymphopenia and subsequent LN or incident proteinuria. Previous studies have found associations between lymphopenia with arthritis and neurological involvement in patients with $\mathrm{SLE}^{22}$. Lymphopenia has also been found to be associated with neuropsychiatric manifestations and disease activity in pediatric patients with $\mathrm{SLE}^{23}$.

Personal non-commercial use only. The Journal of Rheumatology Copyright $\odot$ 2018. All rights reserved. 


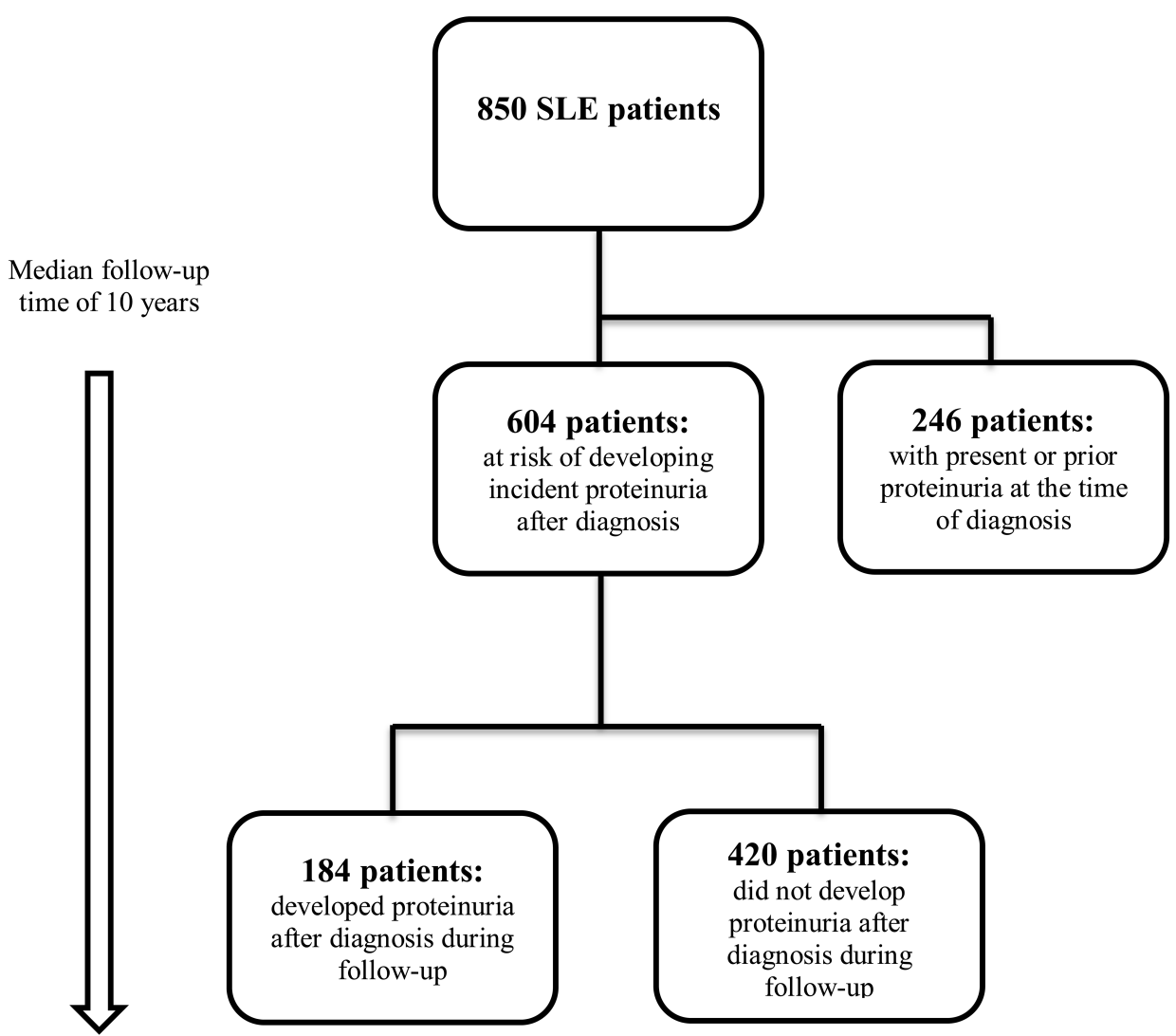

Figure 1. Flowchart illustrating the distribution of proteinuria among the included patients with SLE at the time of diagnosis and during followup. SLE: systemic lupus erythematosus.

It is well known that the prevalence of lymphopenia among patients with SLE differs with regard to age ${ }^{24,25}$. In our study, lymphopenia was found to be associated with the development of incident proteinuria after diagnosis of SLE. This association was still present after adjusting for all significant variables in the univariate analysis, including age, as well as SLE-related medications that could possibly induce lymphopenia. The survival plot (Figure 2) implies that this increased risk is present in both early stages of SLE and throughout the followup time. Our findings are in line with an American study from 2006 that found associations between lymphopenia and several clinical SLE manifestations, including $\mathrm{LN}^{26}$. In addition, several studies have implied an association between increased neutrophil-to-lymphocyte ratio and $\mathrm{LN}^{27,28}$, which may in part be explained by an effect of lymphopenia.

Exactly by which mechanisms lymphopenia is associated with incident proteinuria is not overtly evident, but lymphopenia in SLE is a result of reduced counts of both B and $\mathrm{T}$ lymphocytes ${ }^{29}$, including $\mathrm{CD} 28+\mathrm{T}$ cells ${ }^{30}$. A possible explanation may be that $\mathrm{T}$ cell apoptosis is increased in patients with SLE ${ }^{31,32}$, particularly in those with LN. In addition, a Chinese study from 2014 found higher prevalence of LN among SLE patients with antilymphocyte antibodies, which were also found to be associated with lymphopenia and disease activity ${ }^{33}$. Lymphopenia has also been shown to be associated with disease relapse $\mathrm{e}^{22}$ and can precede flares in $\mathrm{SLE}^{34}$. These and our study findings suggest that lymphopenia could be of prognostic significance regarding the development of incident proteinuria in SLE and thereby potentially also $\mathrm{LN}$.

A history of psychosis was found to be associated with the development of incident proteinuria after SLE diagnosis, and this was particularly pronounced during early years of SLE. Although the observation is of interest, the low number of patients with SLE having psychosis within the analyzed cohort should prompt caution of a potentially spurious finding.

Antimalarial usage was found to be protective against the development of incident proteinuria in the univariate analysis and almost significant in one of the multivariate analyses. A review from 2011 assessing the role of antimalarial agents in the treatment of SLE and LN concluded that antimalarial usage could lead to reduced risk of $\mathrm{LN}$ among patients with SLE as well as reduced risk of flares and certain histological subsets of nephritis among patients with $\mathrm{LN}^{35}$. Unfortunately, the only available data regarding medication usage were the dates of the first prescription. Information regarding cessation

Personal non-commercial use only. The Journal of Rheumatology Copyright (c) 2018. All rights reserved. 
Table 2. Cox regression analyses of the association between demographic and clinical variables at the time of classification with subsequent development of proteinuria of SLE patients $(n=604)$ with no prior or manifest proteinuria at the time of diagnosis.

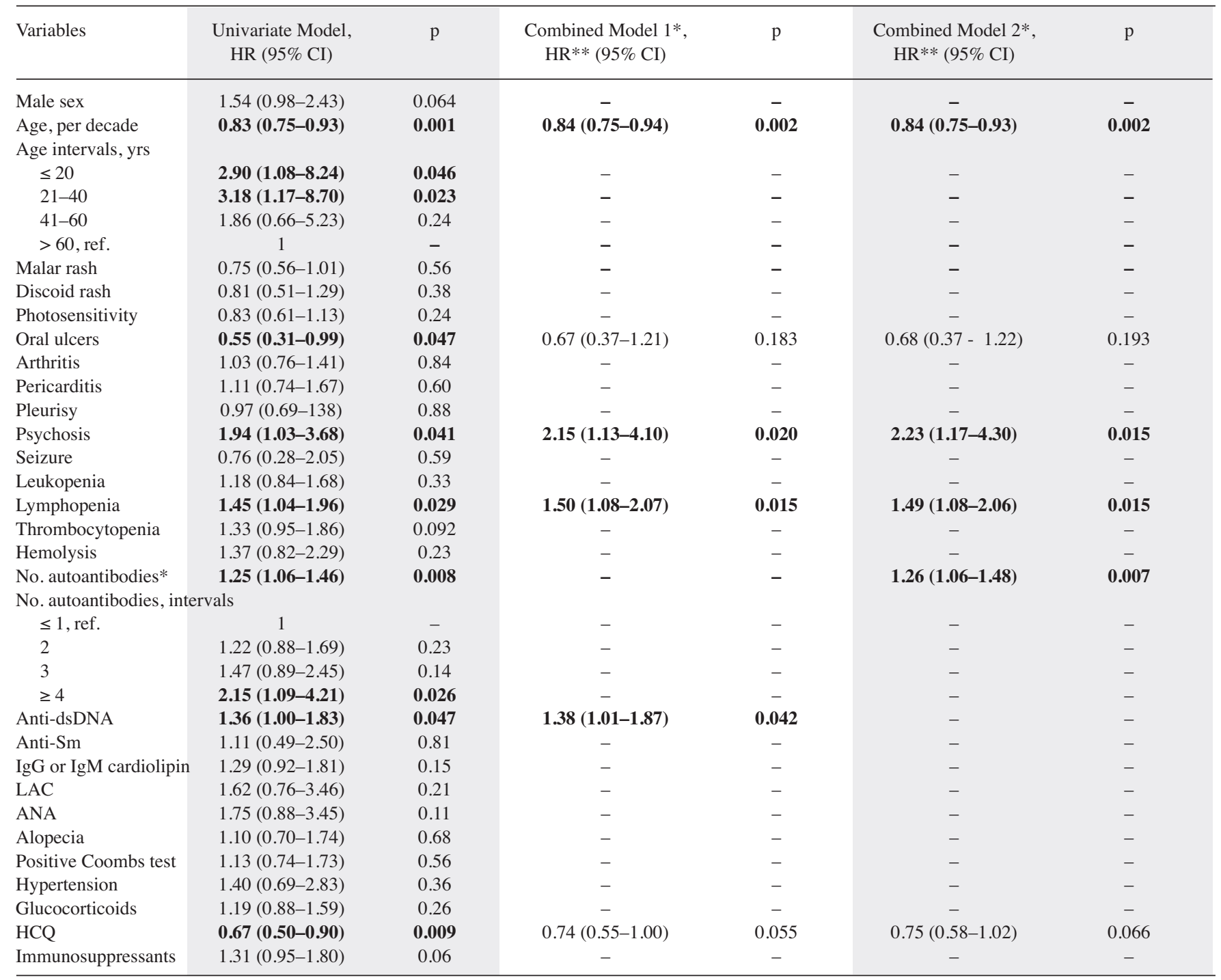

Values in bold face are statistically significant. * Adjusted for variables significantly associated in univariate analyses, selecting anti-dsDNA for model 1 and number of autoantibodies for model 2 because of redundant information in these 2 serological variables. **ANA, anti-dsDNA antibodies, anti-Sm antibodies, IgG and/or IgM cardiolipin antibodies and lupus anticoagulant. SLE: systemic lupus erythematosus; ANA: antinuclear antibodies; IgG: immunoglobulin G; LAC: lupus anticoagulant; HCQ: hydroxychloroquine.

of medication, dosage, and overall compliance was not available and it was thus not possible to examine antimalarial usage as a function of exposure time.

One weakness of our study was that incident proteinuria in the context of a patient with SLE was used as a surrogate for $\mathrm{LN}$ because histological confirmation of LN was not available for all patients with proteinuria. Although histological confirmation is internationally recommended, renal biopsy is for various reasons typically performed in only half of SLE patients with suspected renal disease ${ }^{36}$. Thus we chose in our study to use incident proteinuria as a proxy for $\mathrm{LN}$. Moreover, the analyses of laboratory exposure variables were based only on first-time occurrences and not adjusted for any fluctuations over time, which could be of importance. To study any potential biomarker role, further studies with multiple measurements are warranted. Another potential weakness, given the retrospective features of the study, is the risk of confounding by indication regarding analysis of medications as exposure variables. For the same reason, we have not further explored the role of medications in our study.

The strengths of our study lie within its relatively large cohort size, comprehensive data on the clinical phenotype of the patients, and a long followup time, allowing the use of survival analyses.

The presence of lymphopenia and psychosis, anti-dsDNA antibodies, young age, and a high number of autoantibodies 


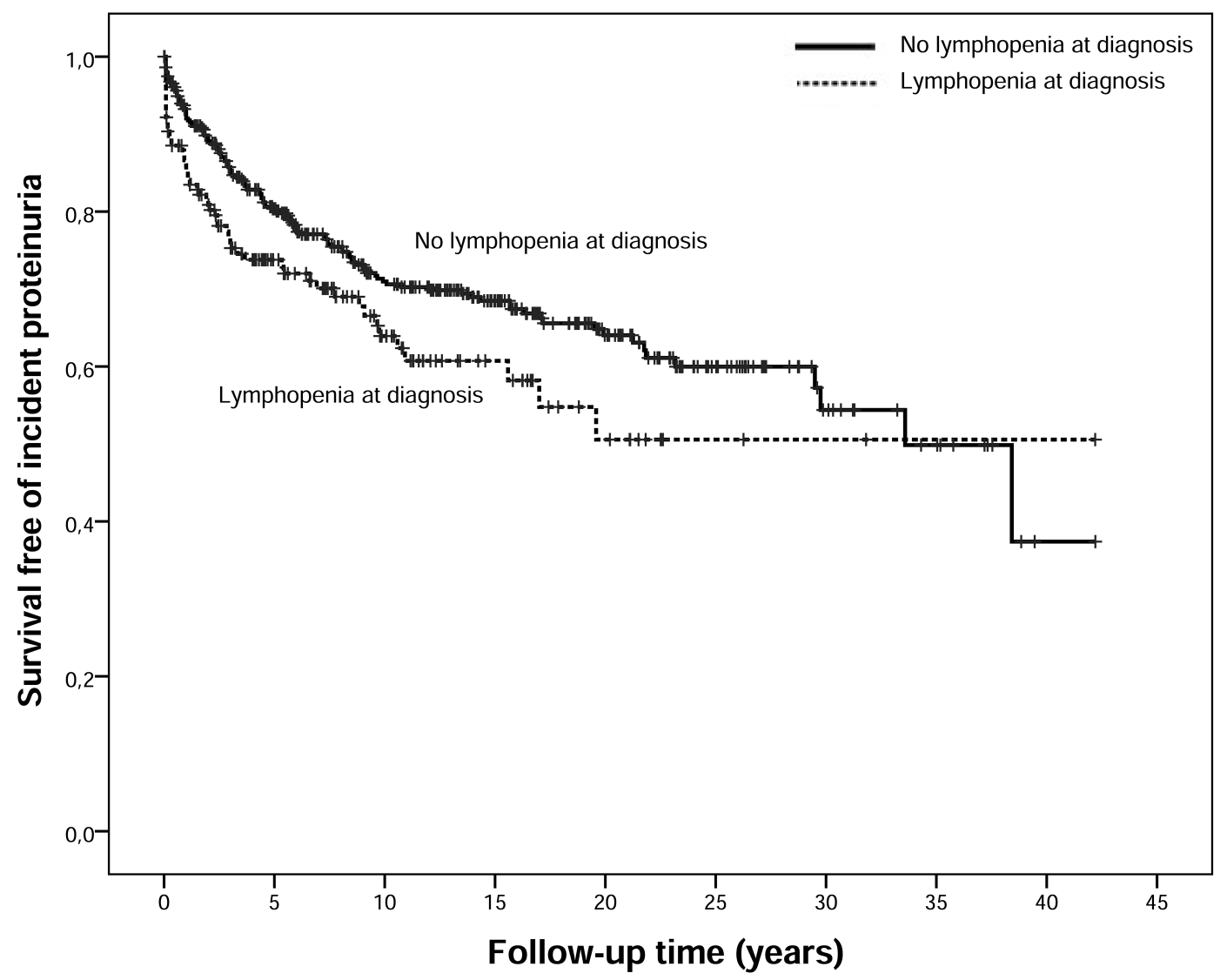

Figure 2. Kaplan-Meier plot of survival free of LN in 604 patients with SLE, with no prior or manifested proteinuria at the time of diagnosis, by the presence or absence of lymphopenia at the time of diagnosis. LN: lupus nephritis; SLE: systemic lupus erythematosus.

at the time of diagnosis were found to be associated with the development of incident proteinuria after SLE diagnosis. Although further replication and studies including multiple measurements are warranted, our study implies that select clinical and serological markers may serve as biomarkers for incident proteinuria in patients with SLE.

\section{REFERENCES}

1. Rahman A, Isenberg DA. Systemic lupus erythematosus. N Engl J Med 2008;358:929-39.

2. Lorenz G, Desai J, Anders HJ. Lupus nephritis: update on mechanisms of systemic autoimmunity and kidney immunopathology. Curr Opin Nephrol Hypertens 2014;23:211-7.

3. Hsu CY, Chiu WC, Yang TS, Chen CJ, Chen YC, Lai HM, et al. Age- and gender-related long-term renal outcome in patients with lupus nephritis. Lupus 2011;20:1135-41.

4. de Carvalho JF, do Nascimento AP, Testagrossa LA, Barros RT, Bonfa E. Male gender results in more severe lupus nephritis. Rheumatol Int 2010;30:1311-5.

5. Livingston B, Bonner A, Pope J. Differences in clinical manifestations between childhood-onset lupus and adult-onset lupus: a meta-analysis. Lupus 2011;20:1345-55.

6. Tomic-Lucic A, Petrovic R, Radak-Perovic M, Milovanovic D, Milovanovic J, Zivanovic S, et al. Late-onset systemic lupus erythematosus: clinical features, course, and prognosis. Clin Rheumatol 2013;32:1053-8.
7. Peschken CA, Katz SJ, Silverman E, Pope JE, Fortin PR, Pineau C, et al; Canadian Network for Improved Outcomes in Systemic Lupus Erythematosus (CaNIOS). The 1000 Canadian faces of lupus: determinants of disease outcome in a large multiethnic cohort. J Rheumatol 2009;36:1200-8.

8. Pons-Estel GJ, Alarcon GS, Hachuel L, Boggio G, Wojdyla D, Pascual-Ramos V, et al; GLADEL. Anti-malarials exert a protective effect while Mestizo patients are at increased risk of developing SLE renal disease: data from a Latin-American cohort. Rheumatology 2012;51:1293-8.

9. Lewis MJ, Jawad AS. The effect of ethnicity and genetic ancestry on the epidemiology, clinical features and outcome of systemic lupus erythematosus. Rheumatology 2017;56:i67-i77.

10. Duarte-García A, Barr E, Magder L, Petri M. Predictors of incident proteinuria among patients with SLE. Lupus Sci Med 2017;4:e000200.

11. Jacobsen S, Petersen J, Ullman S, Junker P, Voss A, Rasmussen JM, et al. A multicentre study of 513 Danish patients with systemic lupus erythematosus. I. Disease manifestations and analyses of clinical subsets. Clin Rheumatol 1998;17:468-77.

12. Tan EM, Cohen AS, Fries JF, Masi AT, McShane DJ, Rothfield NF, et al. The 1982 revised criteria for the classification of systemic lupus erythematosus. Arthritis Rheum 1982;25:1271-7.

13. Hochberg MC. Updating the American College of Rheumatology revised criteria for the classification of systemic lupus erythematosus. Arthritis Rheum 1997;40:1725.

14. Hetland ML. DANBIO - powerful research database and electronic patient record. Rheumatology 2011;50:69-77.

Personal non-commercial use only. The Journal of Rheumatology Copyright $\odot$ 2018. All rights reserved. 


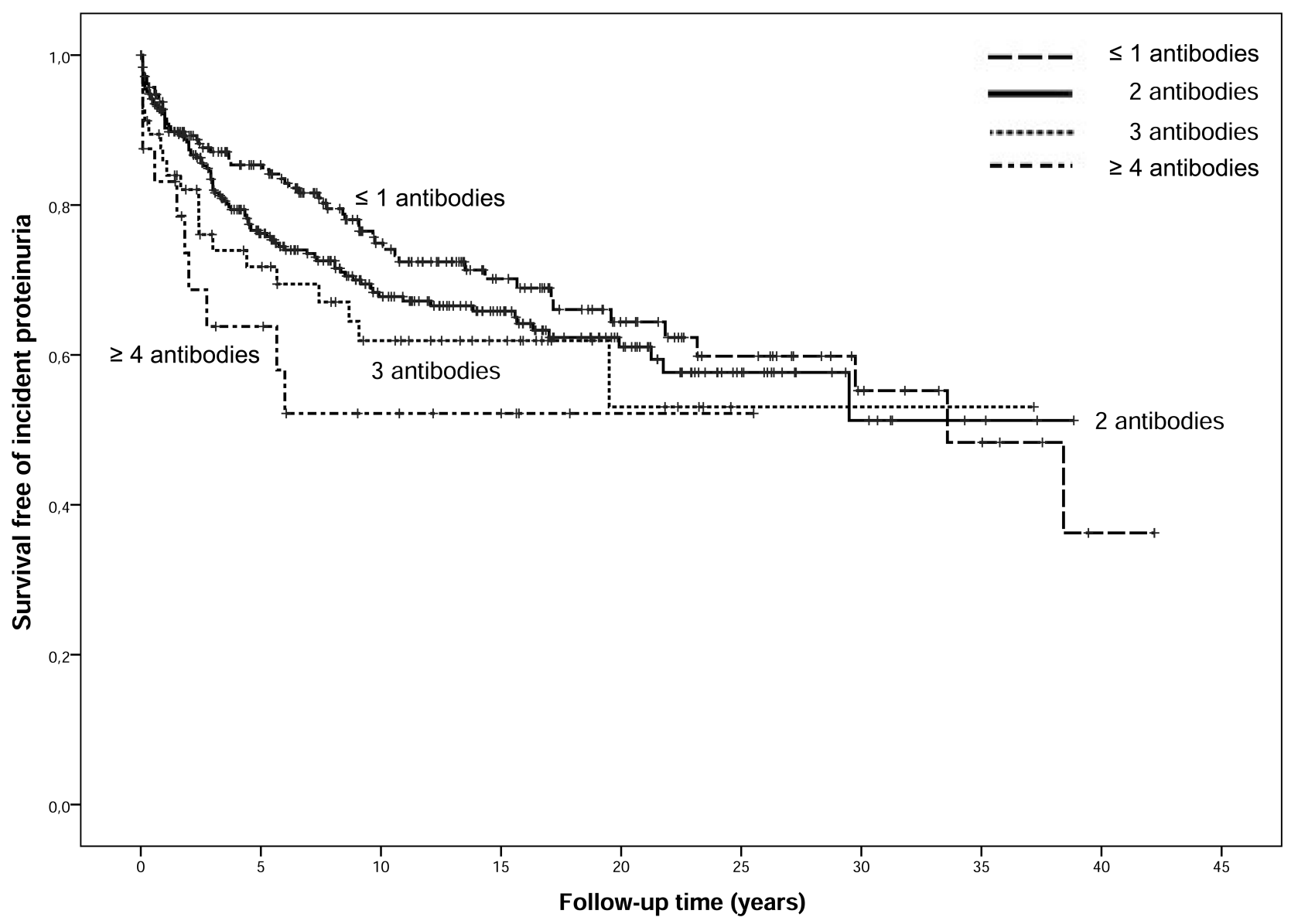

Figure 3. Kaplan-Meier plot of survival free of proteinuria in 604 patients with SLE, with no prior or manifested proteinuria at the time of diagnosis, by number of autoantibodies at the time of diagnosis. SLE: systemic lupus erythematosus.

15. Petri M, Orbai AM, Alarcon GS, Gordon C, Merrill JT, Fortin PR, et al. Derivation and validation of the Systemic Lupus International Collaborating Clinics classification criteria for systemic lupus erythematosus. Arthritis Rheum 2012;64:2677-86.

16. Bastian HM, Roseman JM, McGwin G Jr., Alarcon GS, Friedman AW, Fessler BJ, et al; LUMINA Study Group. LUpus in MInority populations: NAture vs nurture. Systemic lupus erythematosus in three ethnic groups. XII. Risk factors for lupus nephritis after diagnosis. Lupus 2002;11:152-60.

17. Farid EM, Hassan AB, Abalkhail AA, El-Agroudy AE, Arrayed SA, Al-Ghareeb SM. Immunological aspects of biopsy-proven lupus nephritis in Bahraini patients with systemic lupus erythematosus. Saudi J Kidney Dis Transpl 2013;24:1271-9.

18. To $\mathrm{CH}$, Petri M. Is antibody clustering predictive of clinical subsets and damage in systemic lupus erythematosus? Arthritis Rheum 2005;52:4003-10.

19. Tang S, Lui SL, Lai KN. Pathogenesis of lupus nephritis: an update. Nephrology 2005;10:174-9.

20. Yung S, Chan TM. Autoantibodies and resident renal cells in the pathogenesis of lupus nephritis: getting to know the unknown. Clin Dev Immunol 2012;2012:139365.

21. Seret G, Le Meur Y, Renaudineau Y, Youinou P. Mesangial cell-specific antibodies are central to the pathogenesis of lupus nephritis. Clin Dev Immunol 2012;2012:579670.

22. Rivero SJ, Diaz-Jouanen E, Alarcon-Segovia D. Lymphopenia in systemic lupus erythematosus. Clinical, diagnostic, and prognostic significance. Arthritis Rheum 1978;21:295-305.
23. Yu HH, Wang LC, Lee JH, Lee CC, Yang YH, Chiang BL. Lymphopenia is associated with neuropsychiatric manifestations and disease activity in paediatric systemic lupus erythematosus patients. Rheumatology 2007;46:1492-4.

24. Ward MM, Studenski S. Age associated clinical manifestations of systemic lupus erythematosus: a multivariate regression analysis. J Rheumatol 1990;17:476-81.

25. Cooper GS, Parks CG, Treadwell EL, St Clair EW, Gilkeson GS, Cohen PL, et al. Differences by race, sex and age in the clinical and immunologic features of recently diagnosed systemic lupus erythematosus patients in the southeastern United States. Lupus 2002;11:161-7.

26. Vila LM, Alarcon GS, McGwin G Jr., Bastian HM, Fessler BJ, Reveille JD; Lumina Study Group. Systemic lupus erythematosus in a multiethnic US cohort, XXXVII: association of lymphopenia with clinical manifestations, serologic abnormalities, disease activity, and damage accrual. Arthritis Rheum 2006;55:799-806.

27. Li L, Xia Y, Chen C, Cheng P, Peng C. Neutrophil-lymphocyte ratio in systemic lupus erythematosus disease: a retrospective study. Int $\mathrm{J}$ Clin Exp Med 2015;8:11026-31.

28. Qin B, Ma N, Tang Q, Wei T, Yang M, Fu H, et al. Neutrophil to lymphocyte ratio (NLR) and platelet to lymphocyte ratio (PLR) were useful markers in assessment of inflammatory response and disease activity in SLE patients. Mod Rheumatol 2016;26:372-6.

29. Bhuyan UN, Malaviya AN. Deficiency of T cells in blood and tissues and severity of infections in systemic lupus erythematosus. Indian J Med Res 1978;67:269-78. 
30. Kaneko H, Saito K, Hashimoto H, Yagita H, Okumura K, Azuma M. Preferential elimination of CD28+ T cells in systemic lupus erythematosus (SLE) and the relation with activation-induced apoptosis. Clin Exp Immunol 1996;106:218-29.

31. Emlen W, Niebur J, Kadera R. Accelerated in vitro apoptosis of lymphocytes from patients with systemic lupus erythematosus. J Immunol 1994;152:3685-92.

32. Silvestris F, Grinello D, Tucci M, Cafforio P, Dammacco F. Enhancement of $\mathrm{T}$ cell apoptosis correlates with increased serum levels of soluble Fas (CD95/Apo-1) in active lupus. Lupus 2003;12:8-14.

33. Li C, Mu R, Lu XY, He J, Jia RL, Li ZG. Antilymphocyte antibodies in systemic lupus erythematosus: association with disease activity and lymphopenia. J Immunol Res 2014;2014:672126.

34. Mirzayan MJ, Schmidt RE, Witte T. Prognostic parameters for flare in systemic lupus erythematosus. Rheumatology 2000;39:1316-9.

35. Lee SJ, Silverman E, Bargman JM. The role of antimalarial agents in the treatment of SLE and lupus nephritis. Nat Rev Nephrol 2011;7:718-29.

36. Hanly JG, O'Keeffe AG, Su L, Urowitz MB, Romero-Diaz J, Gordon C, et al. The frequency and outcome of lupus nephritis: results from an international inception cohort study. Rheumatology 2016;55:252-62. 